15

\title{
Новый метод исследования структуры сигналов ядерного магнитного резонанса, регистрируемых с использованием модуляционной методики
}

\author{
(C) В.В. Давыдов, ${ }^{1}$ Н.С. Мязин, ${ }^{1}$ C.С. Макеев, ${ }^{1}$ В.И. Дудкин ${ }^{2}$ \\ ${ }^{1}$ Санкт-Петербургский политехнический университет Петра Великого, \\ 195251 Санкт-Петербург, Россия \\ ${ }^{2}$ Санкт-Петербургский государственный университет телекоммуникаций им. проф. М.А. Бонч-Бруевича, \\ 193232 Санкт-Петербург, Россия \\ e-mail: davydov_vadim66@mail.ru
}

Поступило в Редакцию 31 января 2020 г.

В окончательной редакции 2 марта 2020 г.

Принято к публикации 3 марта 2020 г.

Обоснована необходимость исследования структуры сигнала ядерного магнитного резонанса, регистрируемого с использованием модуляционной методики. Установлены особенности регистрации сигнала ядерного магнитного резонанса (ЯМР) с использованием модуляционной методики, зависящие от свойств конденсированной среды, находящейся как в текущем, так и в стационарном состоянии. Предложен новый метод определения вкладов сигналов поглощения и дисперсии в регистрируемый сигнал ЯМР. Представлены результаты экспериментальных исследований.

Ключевые слова: ядерный магнитный резонанс, конденсированная среда, поглощение, дисперсия, амплитудный и фазовый спектр.

DOI: $10.21883 /$ JTF.2020.08.49551.40-20

\section{Введение}

Приборы, принцип работы которых основан на явлении ядерного магнитного резонанса (ЯМР) в настоящее время являются одними из наиболее востребованных для проведения различных исследований в физике, биологии, химии и т.д. [1-6]. В отличие от других методов исследования конденсированных сред измерения, проводимые с использованием ЯМР, не вносят необратимых изменений в физическую структуру и химический состав исследуемой среды [7-11]. Особое место в ЯМР занимают разделы, связанные с контролем состояния конденсированных сред (экспресс-методами), находящихся как в стационарном состоянии, так и текущем [1,2,8,11-13]. Последний - в настоящее время получил новое развитие в связи с востребованностью ЯМР магнитометров на текущей жидкости для измерения параметров сильных и слабых неоднородных магнитных полей, особенно в сложных условиях [14-16]. В малогабаритных ЯМР спектрометрах, расходомерах - релаксометрах и магнетометрах на текущей жидкости сигнал ЯМР регистрируется автодинным детектором с использованием модуляционной методики [3,11-16] и представляет (по форме) непериодическое колебание в виде затухающих по амплитуде пиков („виглей“). Данное колебание повторяется через каждые полпериода модуляции постоянного магнитного поля, в котором находится катушка регистрации. Необходимо отметить, что при исследовании текущей жидкости, особенно турбулентных потоков, использовать другие методы регистрации сигнала ЯМР невозможно.

Для обеспечения высокой точности измерения расхода жидкости q, констант релаксации $T_{1}$ и $T_{2}$, параметров магнитного поля, спектральных компонент система регистрации сигналя ЯМР при использовании модуляционной методики настраивается на максимальное отношение сигнал/шум $(S / N)$. Максимальное значение $S / N$ соответствует резонансным частотам ядер, от которых регистрируется сигнал ЯМР. При отстройке частоты регистрации сигнала ЯМР от резонансной частоты ядер, как это делается в ЯМР спектрометрах высокого разрешения, проводить измерения нецелесообразно $[9,10,17]$. Уровень генерации (значение поля $H_{1}$ ) в автодинном детекторе $[1,11-13,15,16]$, также настраивается на максимальное отношение $S / N$. При такой настройке, регистрируемый автодинным детектором, сигнал ЯМР состоит из сигналов поглощения и дисперсии. Их вклады в регистрируемый сигнал ЯМР изменяется в зависимости от условий эксперимента и состояния среды $[17,18]$.

При проведении исследований механизмов релаксации конденсированных сред, особенно в текущей жидкости, а также для изготовления сред с заданными значениями $T_{1}$ и $T_{2}$ для решения различных задач $[5,11,12]$, отсутствие данных о соотношении между сигналами поглощения и дисперсии в регистрируемом сигнале ЯМР, создают ряд проблем, например, при разработке математической модели для адекватного описания движения вектора ядерной намагниченности в соответствии с проводимым экспериментом или обработки полученных данных. 
Поэтому целью работы является разработка метода для исследования структуры линии регистрируемого сигнала ЯМР от конденсированной среды с использованием модуляционной методики, и определения соотношений между вкладами сигналов поглощения и дисперсии при его формировании от различных условий эксперимента, состава среды и т.д.

\section{Новый метод исследования структуры линии сигнала ядерного магнитного резонанса на основе спектрального анализа}

Проведенные нами исследования $[17,18]$ позволили установить, что с учетом особенностей работы автодинного детектора как интегрального прибора $[1,11,13,15-$ $18]$ форма линии $G(t)$, регистрируемого сигнала ЯМР, может быть описана следующим соотношением:

$$
G(t)=F\left(\frac{A}{A+B} v^{2}(t)+\frac{B}{A+B} U^{2}(t)\right)^{1 / 2},
$$

где $v(t), u(t)$ - сигналы поглощения и дисперсии, $A$ и $B$ - коэффициенты, определяющие вклад в регистрируемый сигнал ЯМР от сигналов поглощения и дисперсии, а $F$ - коэффициент, учитывающий изменения фазы.

Сигналы поглощения и дисперсии для (1) получаются из решения уравнений Блоха, записанных во вращающейся системе координат [19-22]:

$$
\begin{gathered}
\frac{d u(t)}{d t}+\frac{u(t)}{T_{2}}+\Delta \omega v(t)=0, \\
\frac{d v(t)}{d t}+\frac{v(t)}{T_{2}}-\Delta \omega u(t)+\gamma H_{1} M_{z}(t)=0, \\
\frac{d M_{z}(t)}{d t}+\frac{M_{z}(t)}{T_{1}}-\frac{M}{T_{1}}-\gamma H_{1} v(t)=0,
\end{gathered}
$$

где $\Delta \omega=\omega_{0}-\omega_{n m r}-$ расстройка частоты регистрации сигнала ЯМР $\omega_{n m r}$ от частоты прецессии ядер, образующих намагниченность $M_{0}$ в поле $H_{a}\left(\omega_{0}=\gamma H_{a}\right), \chi_{0}-$ статическая ядерная магнитная восприимчивость, $t-$ время, $H_{a}$ - напряженность магнитного поля, в котором размещена катушка регистрации сигнала ЯМР.

При использовании автодинного детектора регистрация сигнала ЯМР осуществляется на резонансной частоте $\omega_{0}(\Delta \omega=0)$. В этом случае система уравнений (2) приобретает следующий вид:

$$
\begin{gathered}
\frac{d u(t)}{d t}+\frac{u(t)}{T_{2}}+\gamma H_{m} \sin \left(\omega_{m} t\right) v(t)=0, \\
\frac{d v(t)}{d t}+\frac{v(t)}{T_{2}}-\gamma H_{m} \sin \left(\omega_{m} t\right) u(t)+\gamma H_{1} M_{z}(t)=0, \\
\frac{d M_{z}(t)}{d t}+\frac{M_{z}(t)}{T_{1}}-\frac{M}{T_{1}}=\gamma H_{1} v(t)=0 .
\end{gathered}
$$

Намагниченность $M$ в (3) при использовании для регистрации сигнала ЯМР автодинного детектора необходимо рассматривать для двух случаев состояния жидкости (стационарное и текущее). Для стационарного состояния жидкой среды значение $M$ в (3) записывается следующим образом:

$$
M=\chi_{0}\left(H_{a}+H_{m} \sin \left(\omega_{m} t\right)\right),
$$

где $\omega_{m}$ и $H_{m}$ - частота и амплитуда модулирующего поля.

Для текущей среды выражение (4) становиться более сложным:

$$
\begin{aligned}
M= & M_{f} \exp \left(-t / T_{1}\right) \\
& +\chi_{0}\left(H_{a}+H_{m} \sin \left(\omega_{m} t\right)\right)\left(1-\exp \left(t / T_{1}\right)\right)
\end{aligned}
$$

где $M_{f}$ - значение намагниченности текущей жидкости на входе катушки регистрации сигнала ЯМР.

Кроме процессов намагничивания и размагничивания в текущей жидкости $[1,5,8,13,15]$ в поле $H_{a}$, в котором находится катушка регистрации, необходимо в (5), а также в (3) при решении этой системы уравнений относительно компонент $v(t), u(t)$ и $M_{z}(t)$, учитывать значение расхода жидкости $q$ и объем катушки регистрации сигнала ЯМР $V_{R}$ в спектрометре. Проведенные нами экспериментальные исследования $[11,13]$ позволили установить, что для регистрации сигнала ЯМР от текущей жидкости необходимо выполнение следующего соотношения между частотой модулирующего поля $\omega_{m}$ и временем нахождения жидкости в катушке регистрации ЯМР спектрометра:

$$
2 T_{m}<V_{R} / q
$$

где $T_{m}$ - период модулирующего поля.

Для получения численных величин $v(t), u(t)$ и $M_{z}(t)$ адекватных условиям эксперимента после решения уравнений (3) для текущей жидкости необходимо значения времени $t$ при их вычислении выбирать меньше $V_{R} / q$ (при временах больших $V_{R} / q$, жидкость будет находиться за пределами катушки регистрации).

При таком рассмотрении $M$ в уравнениях Блоха (3) появляется в зависимости от состояния жидкости еще один дополнительный коэффициент (4) или (5), зависящий от времени. При рассмотрении текущей жидкости зависимость коэффициента (5) от времени более сложная по сравнению с (4), кроме того, добавляется ограничение на частоту модулирующего поля (6). Наличие в (3) трех коэффициентов, зависящих от времени, не позволяет получить аналитическое решение для $v(t)$, $u(t)$ и $M_{z}(t)$ по сравнению с $[21,22]$.

Поэтому система уравнений (3) решается численно относительно компонент $v(t), u(t)$ и $M_{z}(t)$ с учетом начальных условий, которые для стационарной жидкой среды следующие: $M_{z}(0)=\chi_{0} H_{a}, v(0)=0, u(0)=0$, для текущей среды $-M_{z}(0)=M_{f}, v(0)=0, u(0)=0$. 

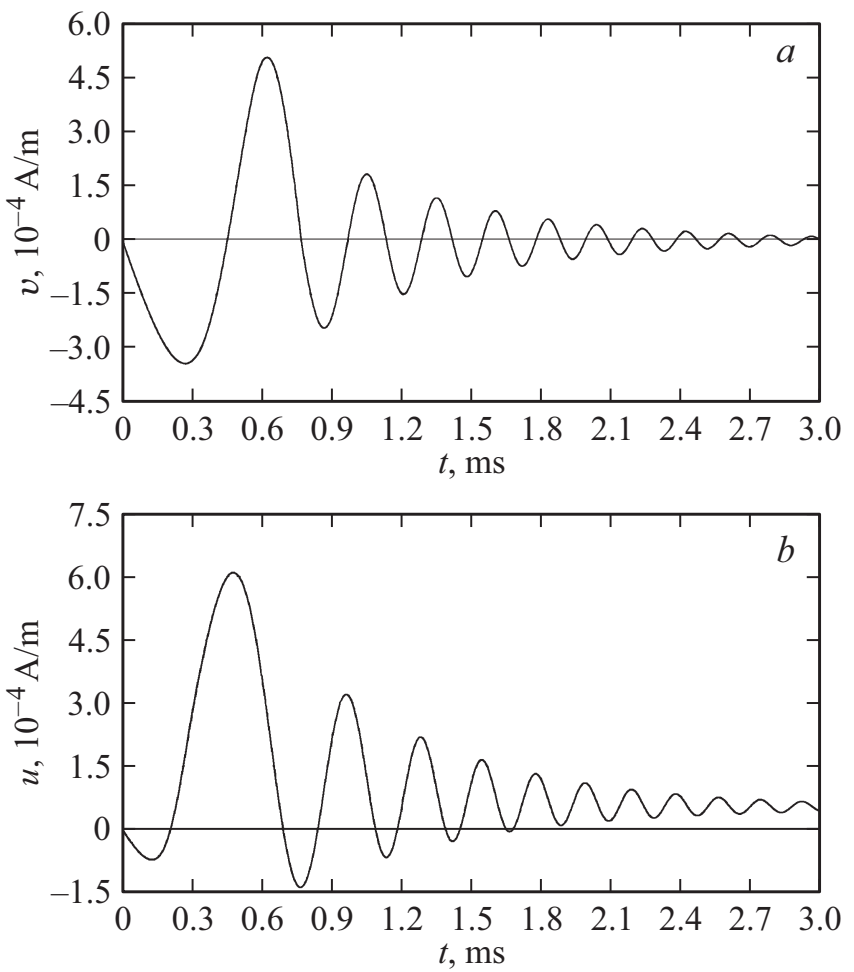

Рис. 1. Расчетные формы линии сигналов ЯМР от воды: $a$ - поглощения; $b-$ дисперсии.

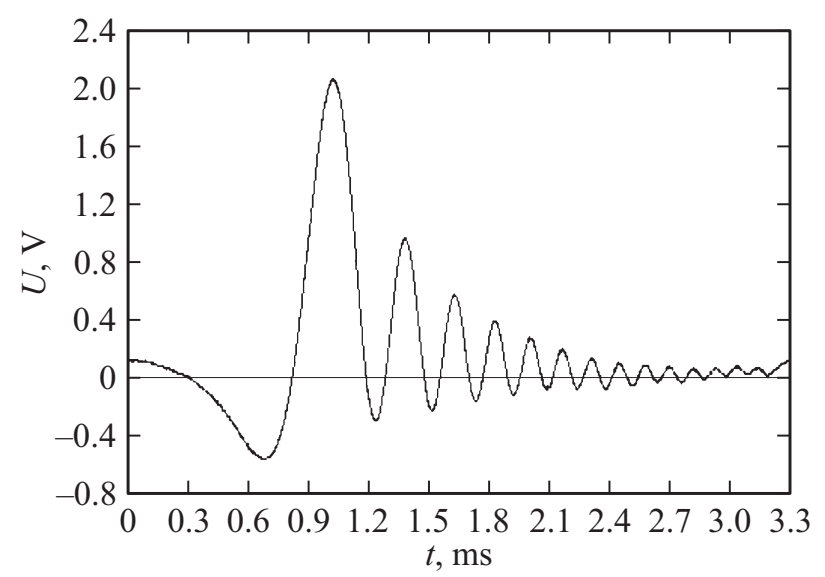

Рис. 2. Зарегистрированный сигнал ЯМР в слабом поле $\left(H_{a}=44563.12 \mathrm{~A} / \mathrm{M}\right)$ с использованием модуляционной методики.

На рис. 1 в качестве примера представлены сигналы поглощения $v(t)$ и дисперсии $u(t)$, полученные при численном решении уравнений (3) при значениях магнитных полей и констант релаксации $T_{1}$ и $T_{2}$, соответствующих эксперименту $\left(T_{1}=1.47 \mathrm{~s} ; T_{2}=1.36 \mathrm{~s}\right.$; $f_{m}=10 \mathrm{~Hz} ; \quad H_{m}=49.33 \mathrm{~A} / \mathrm{m} ; \quad N=6.6 \cdot 10^{29} \mathrm{~m}^{-3}$; $I=1 / 2 ; \quad \mu=1.4 \cdot 10^{-26} \mathrm{~J} / \mathrm{T} ; \quad k=1.38 \cdot 10^{-23} \mathrm{~J} / \mathrm{K}$; $\left.H_{a}=44563.12 \mathrm{~A} / \mathrm{m} ; T=291.8 \mathrm{~K}\right)$.

Анализ полученных на рис. 1 зависимостей показал, что они отражают характер изменения амплитуды ре- гистрируемого сигнала ЯМР в эксперименте (рис. 2), который зарегистрирован спектрометром ЯМР малогабаритной конструкции от стационарной жидкости в слабом магнитном поле. Поэтому нами ранее было предложено использовать для определения вкладов сигналов поглощения и дисперсии соотношение (1), в котором подбирались коэффициенты $A$ и $B$, а также $F$ до получения совпадения формы расчетного сигнала ЯМР с экспериментом $[17,18]$.

Проведенные нами дополнительные расчеты сигнала ЯМР с использованием (1) и (3) позволили выявить ряд проблем. Например, с увеличением $t$ через каждые $T_{m} / 2\left(T_{m}=2 \pi / \omega_{m}\right.$, где $\omega_{m}-$ частота модуляции поля $H_{a}$ ) происходит изменение фазы в форме линии $G(t)$ на $180^{\circ}$ и последовательное уменьшение амплитуды пиков. В эксперименте через каждые полпериода $T_{m} / 2$ поля модуляции $H_{a}$ регистрируется один и тот же сигнал ЯМР. Данное несоответствие теории и эксперимента создает сложности с выбором коэффициента $F(t)$, который зависит от времени. Кроме того, для каждого значения температуры $T$ и состава среды при сравнении экспериментального сигнала ЯМР с $G(t)$ для определения $A$ и $B$ необходимо подбирать каждый раз эмпирически коэффициент $F(t)$. Поэтому возникает необходимость при экспресс-контроле в визуализации сигнала ЯМР. Это создает также дополнительные трудности.

Проведенные нами исследования показали, что использовать для определения $F(t)$ градуировочные зависимости от $T$ нецелесообразно. Многие среды, например, вода обладает различной кислотностью, которая определяется природными условиями. Ее константы релаксации $T_{1}$ и $T_{2}$, которые используются для вычисления сигналов поглощения и дисперсии в (3) будут обладать различной зависимостью от температуры $T$. Значение поля $H_{1}$, особенно при регистрации сигнала ЯМР в слабых полях, при котором получается максимальное отношение $S / N$ для различных состояний среды, также будет обладать различной температурной зависимостью и т.д. Поэтому потребуется очень большое число градуировочных зависимостей $F(t)$ от $T$ как для однокомпонентных сред, так и для многокомпонентных, находящихся как в стационарном, так и в текущем состоянии. Их подготовка и последующая проверка очень трудоемкий процесс ни одного года работы.

Кроме того, при исследовании смесей, если применять (1) для описания формы линии сигнала ЯМР для каждой из компонент смеси, то при их большом количестве в смеси выбор для них $F(t)$ будет крайне трудоемким процессом, который существенно снизит эффективность экспресс-контроля с использованием малогабаритного ЯМР спектрометра [11,12,17].

Поэтому нами предлагается для решения данной проблемы использовать спектральный анализ сигналов поглощения $v(t)$ и дисперсии $u(t)$, полученных из (3). Сопоставляя спектры от рассчитанных сигналов $v(t)$ и $u(t)$ и спектры от экспериментального сигнала, можно 

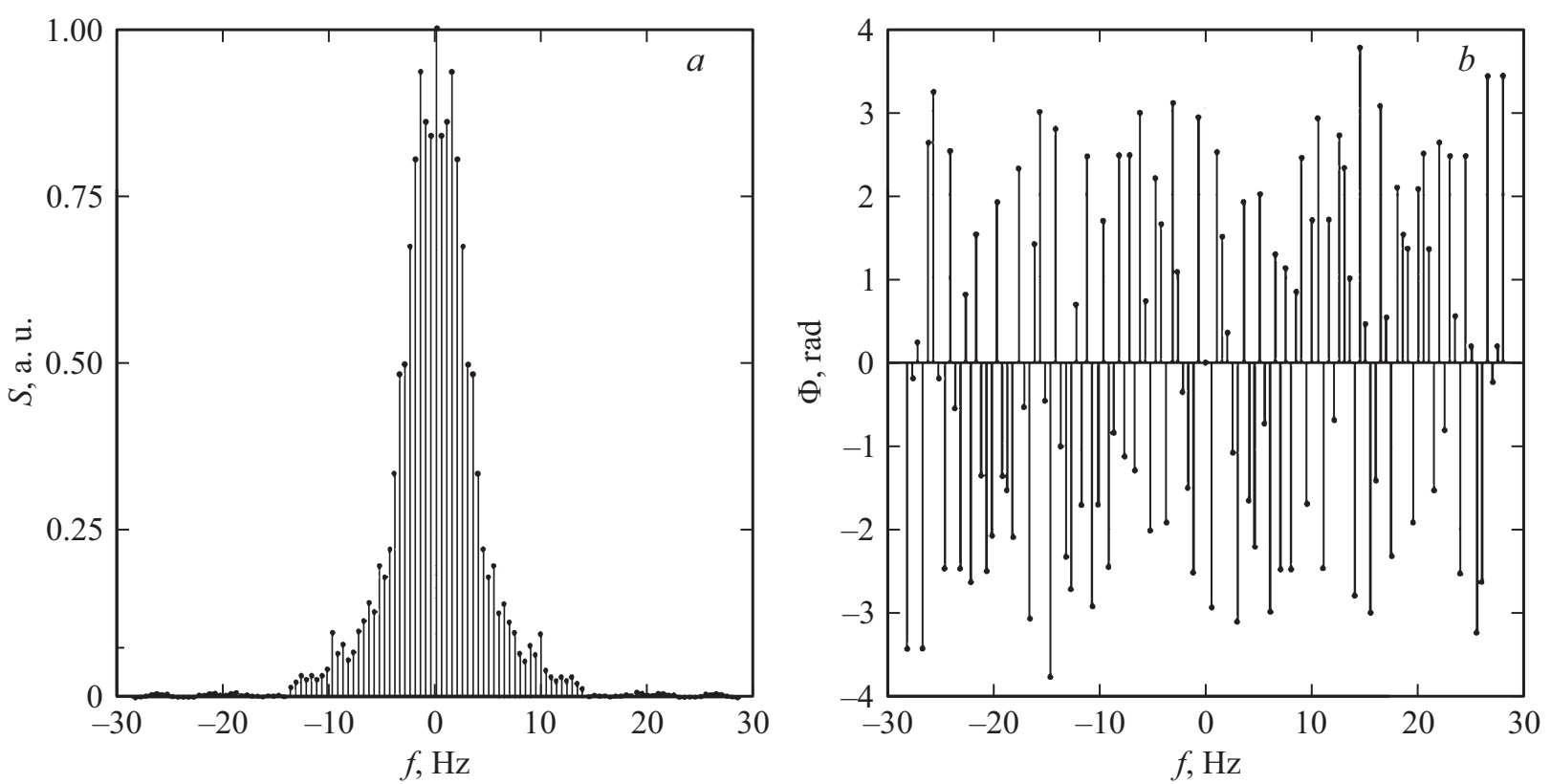

Рис. 3. Спектры расчетной форма линии сигнала ЯМР (поглощение) от воды: $a-$ амплитудный; $b-$ фазовый.
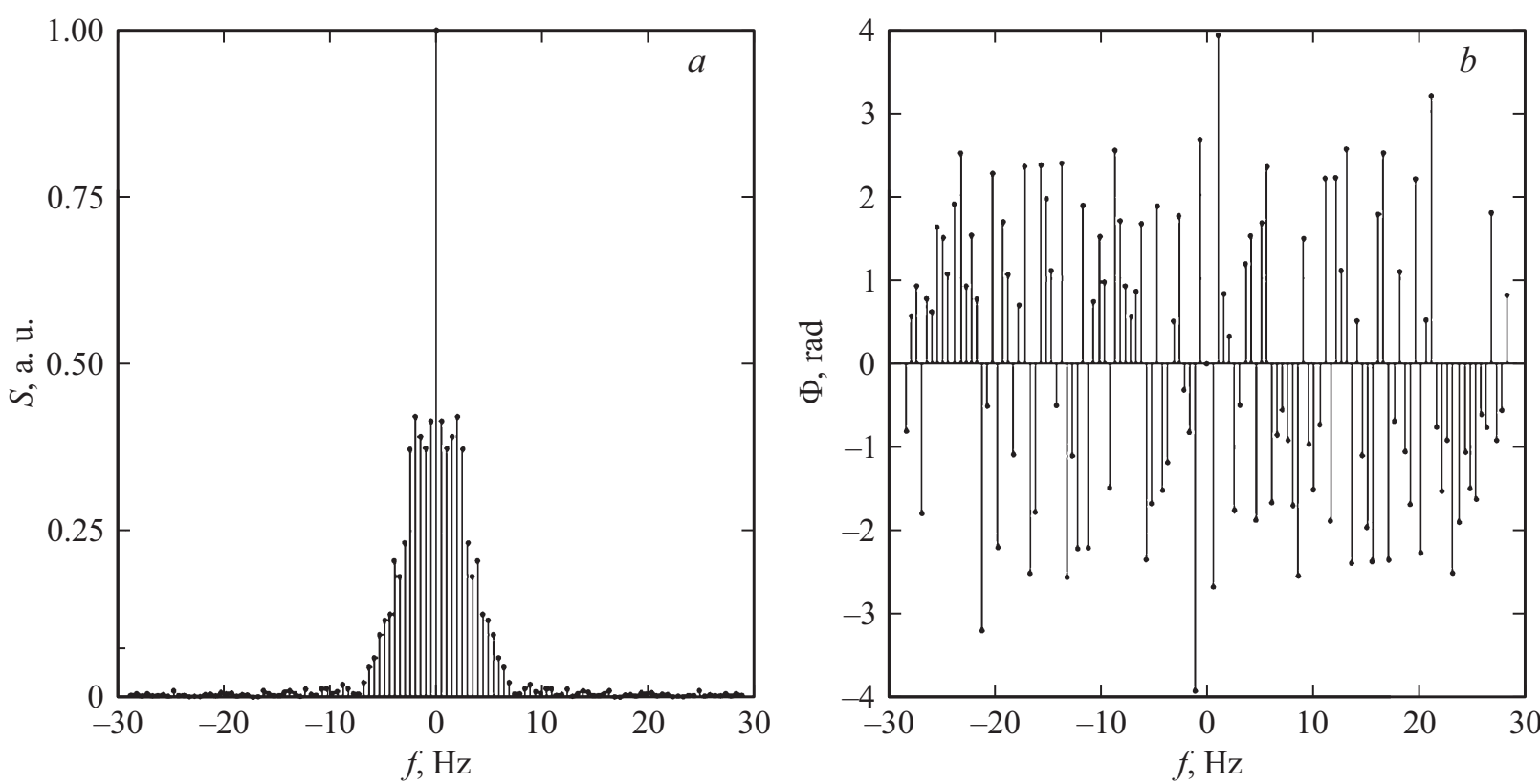

Рис. 4. Спектры расчетной форма линии сигнала ЯМР (дисперсия) от воды: $a-$ амплитудный; $b-$ фазовый.

определить вклады сигналов поглощения и дисперсии в регистрируемый сигнал ЯМР.

\section{Результаты исследования структуры сигнала ЯМР и их обсуждение}

Проведенный нами анализ экспериментальных и рассчитанных сигналов ЯМР (рис. 1 и 2) показал, что их невозможно описать, используя периодические функции. Поэтому наиболее целесообразно для построения их спектров использовать дискретное преобразование Фурье (DFT):

$$
y_{k}=\sum_{n=0}^{N-1} x_{n} e^{-j 2 \pi k n / N},
$$

где $n=0,1,2, \ldots, N-1, x_{n}-$ входная последовательность данных, $N$ - количество элементов входной последовательности данных $x_{n}$.

Гармоники спектра располагаются на оси частот с дискретностью $\Delta f=f_{s} / N$, где $f_{s}$ - частота дискретизации исходной последовательности $x_{n}$. Частота дис- 

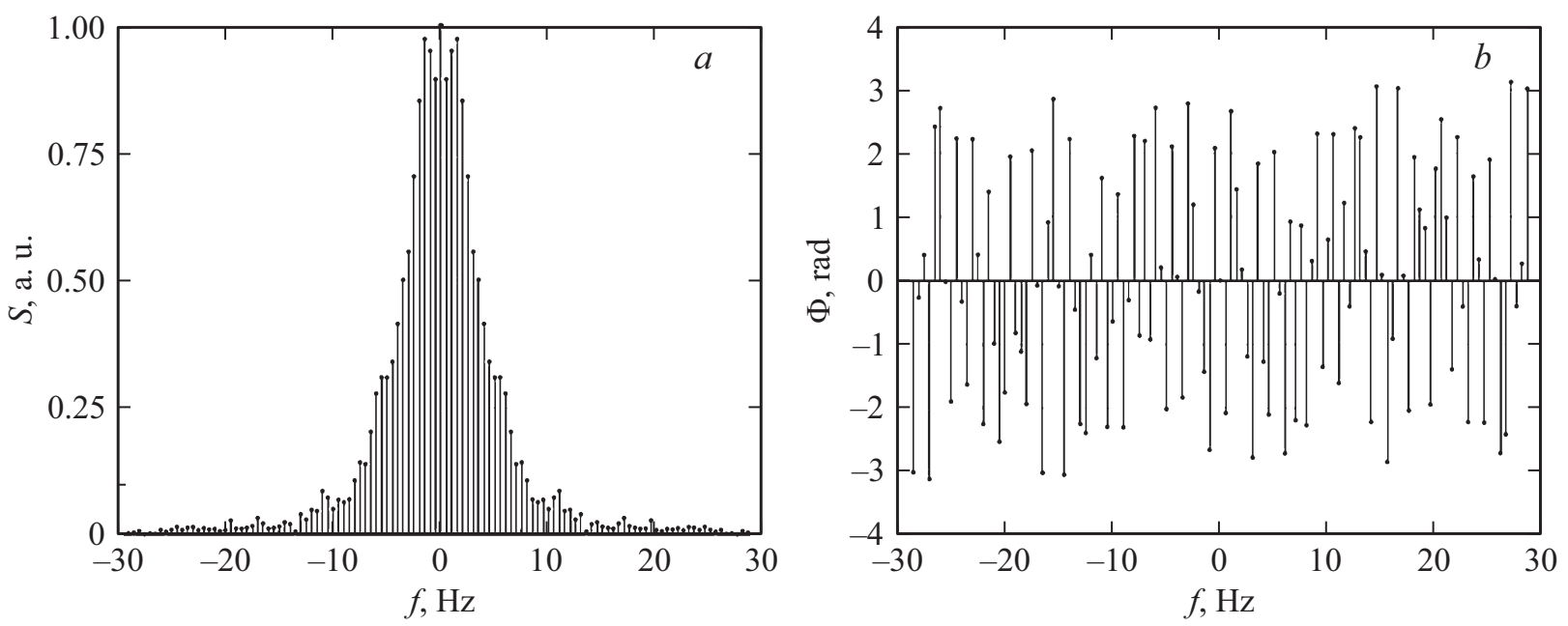

Рис. 5. Спектры экспериментального сигнала ЯМР, зарегистрированного от воды: $a-$ амплитудный; $b-$ фазовый.

кретизации определяется следующим образом. Пусть $\tau$ длительность сигнала ЯМР. Тогда $f_{s}$ можно вычислить, используя следующее соотношение:

$$
f s=N / \tau
$$

В случае, когда $N$ является степенью двойки (например, $N=256=2^{8}$ ), DFT вычисляется по алгоритму FFT (Fast Fourier Transform), который выполняется значительно быстрее DFT и требует меньшее количество вычислительных ресурсов.

Дискретное преобразование Фурье симметрично относительно частоты Найквиста (8), равной $f_{s} / 2$, что позволяет объединить гармоники с номерами $(N / 2-k)$ и $(N / 2+k)$. В результате объединения гармоник можно получить односторонний комплексный спектр с частотами от 0 до $f_{s} / 2$, что соответствует индексам $k=0 \ldots(N / 2-1)$. Масштабированный односторонний комплексный спектр дискретной входной последовательности $x_{n}$ определяется выражением

$$
y_{k}=\left\{\begin{array}{ll}
y_{0} & k=0 \\
\sqrt{2} \frac{y_{k}}{N} & k=1,2, \ldots\left\lfloor\frac{N}{2}-1\right\rfloor
\end{array} .\right.
$$

В соотношении (8) операция в скобках $[N / 2-1]$ означает округление до ближайшего наименьшего целого. Соответственно амплитудный спектр $S(f)=\left|y_{k}\right|$ - модуль одностороннего комплексного спектра, фазовый спектр $\Phi(f)=\arg y_{k}-$ его аргумент, где $f=k \Delta f$.

На рис. 3 и 4 для рассчитанных ранее сигналов поглощения $v(t)$ и дисперсии $u(t)$ (рис. 1) с использованием (8) построены их амплитудные и фазовые спектры. На рис. 5 представлен, полученный с использованием (9), амплитудный и фазовый спектр сигнала ЯМР, зарегистрированного от водопроводной воды (рис. 2) при $T=291.7 \mathrm{~K}$.

Анализ представленных на рис. 3-5 спектров показывает, что в диапазоне изменения частот от -30 до $30 \mathrm{~Hz}$ они обладают одинаковой зависимостью как по амплитуде, так и по фазе. Полученные данные при сравнении амплитуд в амплитудных спектрах $S(f)$ (эксперимент и расчеты), а также значений фазы в фазовых спектрах $\Phi(f)$ (эксперимент и теория) показали, что для гармоник $(k-$ номер гармоники) в этих спектрах, подбирая коэффициенты $A_{1}$ и $A_{2}$, можно с погрешностью не более $1 \%$ получить следующие соотношения:

$$
\begin{gathered}
\Phi_{v}^{\exp }\left(f_{k}\right)=A_{1} \Phi_{v}^{\mathrm{est}}\left(f_{k}\right)+A_{2} \Phi_{u}^{\mathrm{est}}\left(f_{k}\right), \\
S_{v}^{\ni}\left(f_{k}\right)=A_{1} S_{v}^{p}\left(f_{k}\right)+A_{2} S_{u}^{p}\left(f_{k}\right),
\end{gathered}
$$

где индекс ехр - соответствует эксперименту, индекс est - расчету.

В этом случае значения коэффициентов $A_{1}$ и $A_{2}$ отображают вклад сигналов поглощения $v(t)$ и дисперсии $u(t)$ в регистрируемый сигнал ЯМР. При расчете $A_{1}$ и $A_{2}$ в соотношении (10) для амплитудных спектров необходимо учитывать связь между нормированием по амплитуде (рис. $3, a, 4, a$ и 5, $a$ ), так как расчет сигналов поглощения и дисперсии (рис. 1) выполняется для величин, которые измеряются в единицах намагниченности $(\mathrm{A} / \mathrm{m})$, а амплитуда регистрируемого сигнала ЯМР (рис. 2) измеряется в вольтах.

Сравнение полученных нами значений $A_{1}=0.849 \pm$ \pm 0.008 и $A_{2}=0.151 \pm 0.001$ со значениями коэффициентов $A=0.846 \pm 0.016$ и $B=0.154 \pm 0.003$, которые определяют вклады от сигналов поглощения $v(t)$ и дисперсии $u(t)$, полученными с использованием (1), показали, что они совпадают в пределах погрешности измерений. Это подтверждает достоверность предложенного нами метода. Кроме того, результат численного сравнения показывает, что разработанный нами спектральный метод обладает более высокой точностью определения коэффициентов по сравнению с методом определения коэффициентов с использованием (1).

Отметим, что результаты оценки вкладов от сигналов поглощения $v(t)$ и дисперсии $u(t)$ в регистрируемый 
сигнал ЯМР при работе автодинного детектора на срыве колебаний $[1,14]$ предложенным нами методом совпали с результатами, полученными ранее в работах $[18,22]$.

\section{Заключение}

Полученные результаты показывают, что предложенный нами новый метод с использованием спектрального анализа, позволяет получать информацию о вкладах сигналов поглощения $v(t)$ и дисперсии $u(t)$ в регистрируемый сигнал ЯМР с использованием модуляционной методики от конденсированной среды, находящейся как в стационарном, так и в текущем состоянии. Это позволяет проводить исследования структуры линии зарегистрированного сигнала ЯМР от различных параметров конденсированных сред, а также от условий регистрации сигнала ЯМР в различных конструкциях приборов, например, в малогабаритном ЯМР спектрометре при проведении экспресс-контроля [2,11,12] или ЯМР расходомере-релаксометре $[8,13,14]$. Полученные новые данные о состоянии среды с использованием нового метода, например, при экспресс-контроле позволят принимать более обоснованное решение о дальнейших действиях $[2,11,12]$.

Кроме того, использование разработанного нами нового метода позволяет более эффективно проводить фундаментальные научные исследования изменения вкладов сигналов поглощения и дисперсии в структуру регистрируемого сигнала ЯМР от конденсированной среды, находящейся как в стационарном, так и текущем состоянии при изменении ее времен продольной $T_{1}$ и поперечной $T_{2}$ релаксации. Эти исследования необходимы для решения задач, связанных с разработкой новых жидких сред с заданными значениями $T_{1}$ и $T_{2}$ для ЯМР магнитометров на текущей жидкости $[14,15]$.

\section{Конфликт интересов}

Авторы заявляют, что у них нет конфликта интересов.

\section{Список литературы}

[1] Жерновой А.И., Дьяченко С.В. // ЖТФ. 2015. Т. 85. Вып. 4. C. $118-122$.

[2] Давыдов В.В., Мязин Н.С., Дудкин В.И., Гребеникова Н.М. // ЖТФ. 2018. Т. 88. Вып. 12. С. 1885-1889.

[3] Karseev A.Yu., Vologdin V.A. // J. Phys. Conf. Series. 2015. Vol. 643(1). P. 012108.

[4] Иванов М.Ф., Киверин А.Д., Смыгалина А.Е., Зайченко В.М. // ЖТФ. 2018. Т. 88. Вып. 1. С. 147-150.

[5] Давыдов В.В., Дудкин В.И. // ЖТФ. 2016. Т. 86. Вып. 7. C. $154-158$.

[6] Калиничев А.А., Борисов Е.Н., Поволочкий А.В., Иванова Т.Ю., Иванов Д.А. // ЖТФ. 2016. Т. 86. Вып. 9. С. 156158.

[7] Коган В.Т., Антонов А.С., Лебедев Д.С., Власов С.А., Краснюк А.Д. // ЖТФ. 2013. Т. 83. Вып. 3. С. 132-139.
[8] Marusina M.Y., Bazarov B.A., Galaidin P.A., Marusin M.P., Silaev A.A., Zakemovskya E.Y., Mustaev Y.N. // Measurement Techniques. 2014. Vol. 57. N 5. P. 580-587.

[9] Alashkin E.M., Kondratyeva E.T., Kuzmin V.V., Safullin K.R., Stanislavovas A.A., Savinkov A.V., Klochkov A.V., Tagirov M.S. // JEPT Letters. 2018. Vol. 107. N 2. P. $111-118$.

[10] Filippov A.V., Artamonova M.R., Rudakova M.F., Gimatdinov R.G., Skirda V.D. // Magn. Res. Chem. 2012. Vol. 50. N 2. P. 114-119.

[11] Давыдов В.В., Дудкин В.И., Карсеев А.Ю. // ЖПС. 2015. T. 82. № 5. C. 736-742.

[12] Давыдов В.В., Величко Е.Н., Дудкин В.И., Карсеев А.Ю. // ПТЭ. 2015. № 2. С. 72-76.

[13] Давыдов В.В., Дудкин В.И., Мязин Н.С. // Радиотехника и электроника. 2016. Т. 61. № 10. С. 1026-1032.

[14] Давыдов В.В., Дудкин В.И., Карсеев А.Ю. // ЖТФ. 2015. Т. 85. Вып 3. С. 138-143.

[15] Дьяченко С.В., Кондрашкова И.С., Жерновой А.И. // ЖТФ. 2017. Т. 87. Вып. 10. С. 1596-1598.

[16] Жерновой А.И., Комлев А.А., Дьяченко С.В. // ЖТФ. 2016. Т. 86. Вып. 2. С. 146-148.

[17] Myazin N.S., Davydov V.V. // J. Phys. Conf. Series. 2018. Vol. 1135(1). P. 012061.

[18] Давыдов В.В., Мязин Н.С., Логунов С.Е., Фадеенко В.Б. // Письма в ЖТФ. 2018. Т. 44. Вып. 4. С. 50-56.

[19] Bloch F. // Phys. Rev. 1946. Vol. 70. N 7. P. 460-478.

[20] Bloch F., Hansen W.W., Packard F. // Phys. Rev. 1946. Vol. 70. N 7. P. 474-492.

[21] Абрагам А. Ядерный магнетизм. М.: ИЛ, 1963. 612 с.

[22] Леше А. Ядерная индукция. М.: ИЛ, 1963. 684 с. 